EPJ Web of Conferences 101, 06027 (2015)

DOI: $10.1051 /$ epjconf/ 201510106027

(C) Owned by the authors, published by EDP Sciences, 2015

\title{
The period distribution of Cepheids: a test of stellar evolution
}

\author{
M.A.T. Groenewegen ${ }^{1, a}$, L. Girardi ${ }^{2}$, and the VMC consortium \\ 1 Koninklijke Sterrenwacht van België, Ringlaan 3, B-1080, Brussel, Belgium \\ 2 Osservatorio Astronomico di Padova-INAF, Padova, Italy
}

\begin{abstract}
The period distributions of classical Cepheids in the Small and Large Magellanic Cloud are quite different. Using the TRILEGAL population synthesis code and a theoretical instability strip the ultimate aim is to understand these differences quantitatively. First results are presented for one area in the LMC using VMC NIR data.
\end{abstract}

\section{Cepheids and population synthesis}

The period distributions of classical Cepheids in the LMC and SMC are very different, as shown e.g. in figure 9 in Soszyński et al. [1], based on OGLE-III data. The peak in the period distribution of fundamental mode cepheids in the $\mathrm{LMC}$ is at $\log P \sim 0.5$, in the SMC at $\log P \sim 0.15$. The smaller and less massive SMC has more Cepheids (4630) than the LMC (3361). Metallicity is obviously a factor as the IS (instability strip) depends on it, and differences in the Star Formation Histories (SFHs), but, can we understand these differences also quantitatively?

The VISTA Magellanic Cloud Survey (VMC, Cioni et al. [2]) is a survey of about $180 \square^{2}$ in $Y J K$ covering the entire MC system, i.e. SMC, LMC, Bridge and stream. The main scientific aims include the determination of the (spatially resolved) SFH and Age Metallicity Relation (AMR) and to obtain a 3-D view of the MCs through the use of various standard candles. Rubele et al. [3] e.g. derived the SFH and AMR in a few tiles from the deep CMDs, and Ripepi et al. [4] studied the Cepheid population in two tiles. In the latter paper, the known population of OGLE-III Cepheids was correlated with the VMC data, and using the 12 epochs in $K$, light curves and mean magnitudes were derived.

TRILEGAL is a population synthesis code (Girardi et al. [5]) for the Milky Way, but where an external component can be added as well. Given an SFR and AMR, it calculates the magnitudes of all stars in a given line of sight. In combination with an assumed IS and a relation between period and the fundamental stellar parameters, one can build synthetic populations of variable stars.

The theoretical IS and $P\left(T_{\text {eff }}, M, L\right)$ relation for classical fundamental mode Cepheids from Bono et al. [6] have been used. They provide relations for $Z=0.004,0.008$ and 0.02 and a logarithmic interpolation in $\log Z$ has been used. If the synthetic star (with known $[\mathrm{M} / \mathrm{H}]$ ) is inside the (interpolated) instability strip the period is calculated from the relations in Bono et al., and its $K$-magnitude recorded.

The results are shown for the tile LMC 6_6 which has 158 FU Cepheids (Ripepi et al.). The TRILEGAL simulation is for the SFH and AMR derived in Rubele et al. for this tile. From the fitting of the CMD they derived a distance modulus of 18.40. Figure 1 shows the comparison between the observed (filled circles) and synthetic (open circles) $P L$-relation. The model predicts quite some so-called ultra long period (ULP) Cepheids which are not in the observed data. This is due to saturation either in OGLE (the Cepheids in the OGLE shallow survey (Ulaczyk et al. 2013) have not been considered yet), or VMC, which saturates near $K \sim 11.5$ ). The right-hand panel cuts the absolute magnitude at -7 . The synthetic $P L$-relation (the blue solid line) is $M_{\mathrm{K}}=-3.10 \log P-2.62$ while the one found by Ripepi et al. (the black dashed line) is $M_{\mathrm{K}}=-3.30 \log P+16.07$, or a $\mathrm{ZP}$ of $-2.41 \pm 0.03$ for their preferred DM of $18.47 \pm 0.03$.

\footnotetext{
a e-mail: martin.groenewegen@oma.be
} 

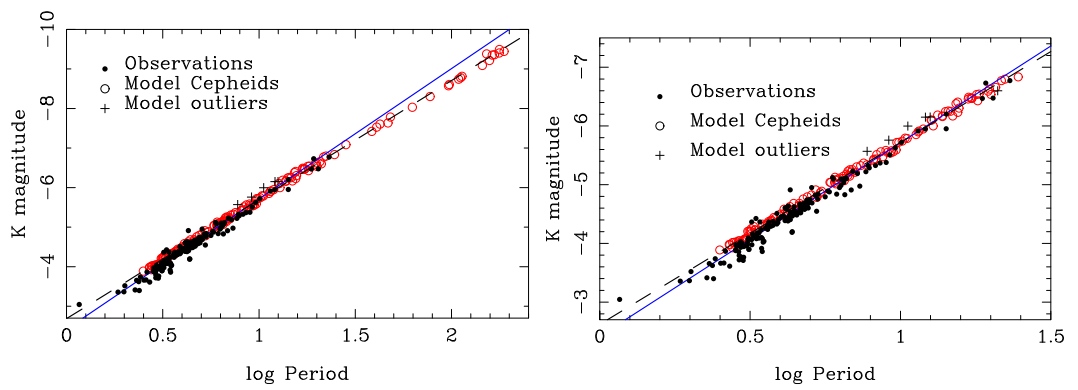

Fig. 1. The comparison between the observed (filled circles) and synthetic (open circles) $P L$ relation, see text.
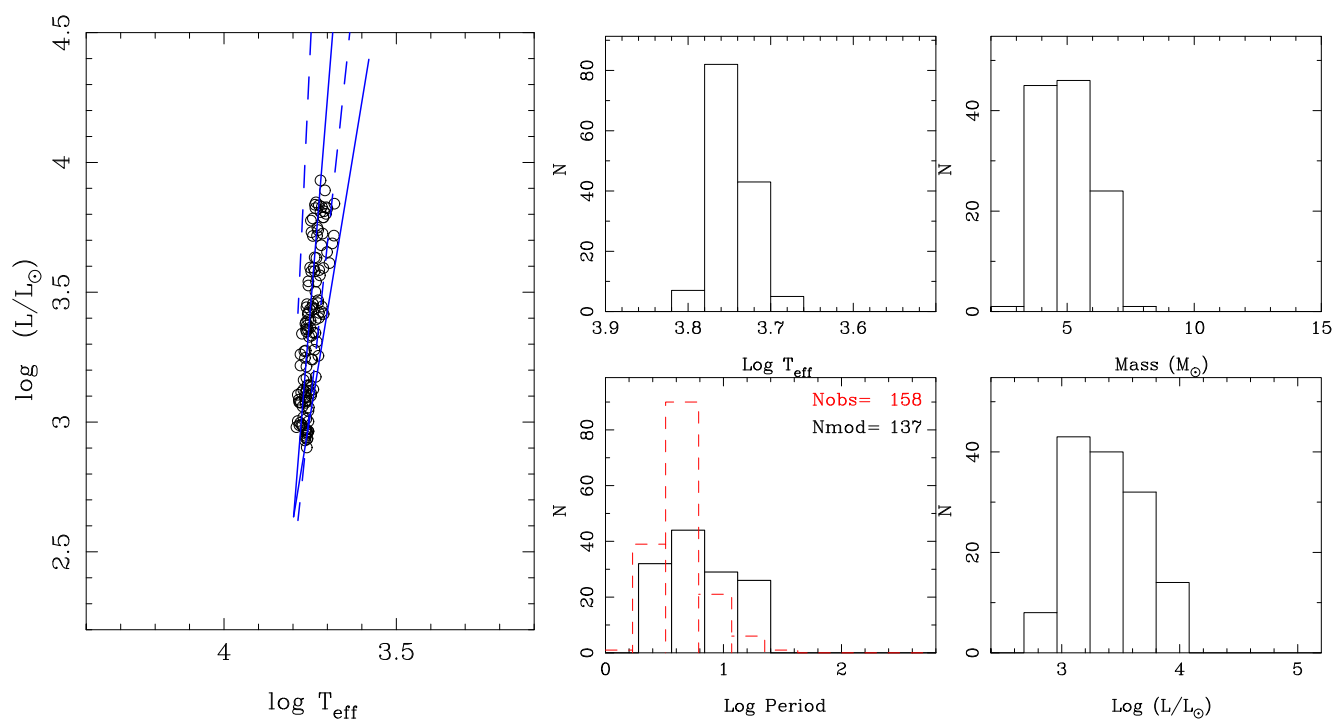

Fig. 2. The synthetic HRD on the left, with the instability strip for $Z=0.02$ (solid line) and $Z=0.004$ (dashed line) indicated. On the right synthetic histograms of effective temperature, mass, period, and luminosity.

Figure 2 shows on the left the synthetic HRD, and on the right the synthetic histograms of some quantities. The number of Cepheids found compares well to the observations but the synthetic model has more longer period Cepheids. The slope and the $\mathrm{ZP}$ of the $P L$-relation differ, but the difference in magnitude is only $\sim 0.1$ for $\log P=0.5$ and essentially 0 for $\log P=1$. For a very first test these results are encouraging. Future work will:

- Study the influence of the location of the IS, and details of the $P\left(T_{\text {eff }}, M, L\right)$ relation.

- Extend the code to first overtone pulsators.

- Ultimately extend this to the entire VMC MC survey area.

- Perform this comparison for Cepheids in the Solar Neighbourhood, to check the procedure for another metallicity and SFH.

- Include other types of variable stars, e.g. RR Lyrae. One could then study the RR Lyrae population in the direction of the Galactic Centre.

\section{References}

1. I. Soszyñski, R. Poleski, A. Udalski, M.K. Szymañski, M. Kubiak et al., AcA 60, 17 (2010)

2. M.R.L. Cioni, G. Clementini, L. Girardi et al., Astron. \& Astrophys. 527, A116 (2011)

3. S. Rubele, L. Kerber, L. Girardi, M.R. Cioni et al., Astron. \& Astrophys. 537, A106 (2012)

4. V. Ripepi, M.I. Moretti, M. Marconi, G. Clementini et al., Mon.Not.RAS 424, 1807 (2012)

5. L. Girardi, M.A.T. Groenewegen, E. Hatziminaoglou et al, Astron. \& Astrophys. 436, 895 (2005)

6. G. Bono, V. Castellani, M. Marconi, Astrophys. J. 529, 293 (2000) 